\title{
Efficient Scale Invariant and Back Propagation Neural Network Method using LIP Region Segmentation
}

\author{
Shaifali Sharma, Geetanjali Babbar, Ishdeep Singla, Gagandeep Jindal
}

\begin{abstract}
With the advent of technological sensor devices and human interface machine technology, there has been extensive research done in lip segmentation methods by several researchers - some linguistic features required for interaction with the machine equipment. Therefore, research work has been done in the audio speech detection scheme for recognition of lip reading. Visual lip reading technology developed based on the extraction of features of the lip. Lip segmentation is an essential approach to recognize lip reading scheme. Meanwhile, it helps to improve parameters. Several methods studied to segment the lip area based on localized active contour method using twice contour finding and combined color-space method. Apply the illumination histogram equalization to real color images to reduce the distortion of uneven illumination. The proposed method implemented can get better accuracy rate and segmentation results and compare with the existing process using area or circle as the region to segment grayscale images and combined in the color-space image. Using SIFT and BPNN, the inner region of the lip found in the result. The experiment tool is used MATLAB 2016 and designs a PROJECT APPLICATION. Improve the success rate and reduce the segmented error and compared with the current metrics. The experimental analysis determines the accuracy rate with 94\%; error rate reduces with Segmented Error $\%$ and Overlap Error rate value with $79.73 \%$.
\end{abstract}

Index Terms: Lip Segmentation, Feature Extraction - Scale Invariant Feature Transformation, BPNN - Back Propagation Neural Network, and Active Contour.

\section{INTRODUCTION}

In the modern world of technology, Graphical lip detection has been done through the association of the equipment visualization and observation of the language [1]. In lip detection, images or the video of the face are captured through equipment and after that features of the mouth are extracted and then recognize the accents of the characteristics of the mouth through detection method [2][3]. Human system interaction, pattern detection, and artificial intelligence have become a common area of research that overcomes the issue of the detection value of speech detection scheme that occurs due to interference or distortion [4]. Picture segmentation is the method of the segmentation of the digital picture into various segments [5]. Lip segmentation is an essential method of the visualization lip detection scheme; exactness of lip segmentation improves the detection value or rate. Segmentation depends on the features like as segmented pixels on area and borders, statistical data, the average weight of the segmented area [6][7]. By the medical theory of China,

Revised Manuscript Received on June 22, 2019.

Shaifali Sharma, CEC, CGC, Landran,mohali, India

Geetanjali Babbar, CEC, CGC, Landran,Mohali, India

Ishdeep Singlaa, CEC, CGC, Landran, Mohali,India.

Gagandeep Jindal,CEC,CGC,Landran,Mohali,India. the color of the lip of an individual is taken as the marks and indicators if the stomach is in good physical shape or not [8]. Therefore, computer technology can determine the relationship between lip color and illness through lip segmentation process [9][10]. Lip segmentation process categorized into two types, which is identified through color or intensity area. The kind of approach utilizes color conversion or color filtration by extending the change among the field of lip and area of non-slip.

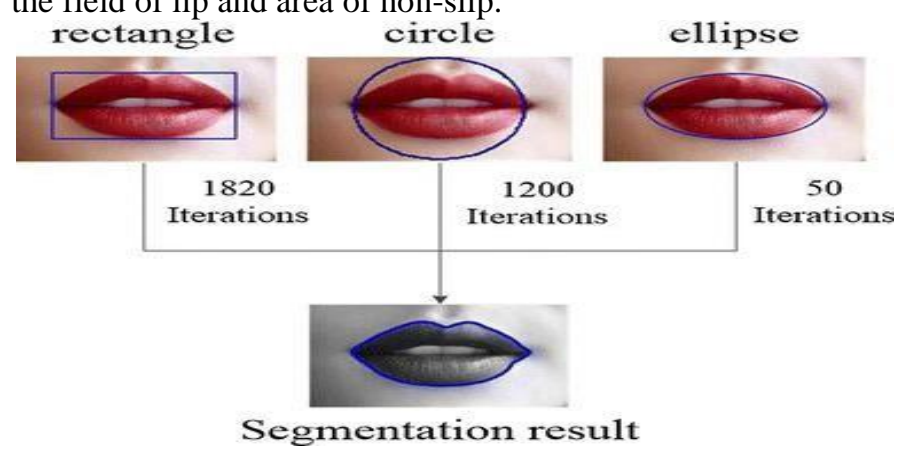

Fig. 1 Lip image Segmentation [13]

Though the lip area identified in less period, it leads to high color distortion and color compact. The other is the modelbased technique like as geometrical method, contour method, live shape method [11][12]. The other is the model with a group of characteristics ideas to appropriate lip contour and withkey features to determine the method of the lip contour. The benefit of this model is of maximum strength. Hence the issue of this technique is the extraction of the features of the lip area in the absence of the semiautomation operator method [13]. Presently lip segmentation is used in various applications areas like as language detection, lip detection, face views, audio recognition, and so forth. The lip segmentation technique may get difficulty in detection of the lip area whenever the color of the lip is not differentiated easily. Traditional active contour model is a popular method of lip segmentation [14]. The Active contour model permits the basic contour for deformation through minimization of the global power value for getting the final segmented image. Active contour model has some benefits that include exactness of the of object borders. Other is getting the smooth image by extraction of features of face image [15].

The existing method is ACM can be classified into two classes:-
1.
Edge Based and
2. Model Based Model

Edge model accepted image gradient as limitation situation, which makes the contour to coverage to object border-line. Studies have implemented several techniques to enhance edge based models. These structures incomplete convergence issue due to the fuzzy set 
and weak object area and noisy image.

Region-based methods are resistant to the noisy image. It uses image statistical data as limitation situation and the performance metric is superior to edge-based models. In these methods, an image can segment based object region in the case of minimum or even without borderline.

Existing LACM technique is using the contour-based region in detection combined color-space image. Illumination histogram equalization method is applied to the real image (RGB image) to optimize the distortion consequence from uneven illumination.

In research work, has implemented a segmentation with SIFT and BPNN (Backpropagation neural network) method. In SIFT (Scale-invariant feature transformation) algorithm used to extract the features based on key-points. It removes the image properties in the form of MATRIX format and read the image color features, which reduce the matrix of the real image in BPNN classifier method to train the feature vector and test the region based segmented boundary area. BPNN method used to classify the feature set and passed into three layers based on, (i) Input Layer (ii) hidden Layer and (iii) Output Layer.

This model used to simulate function to filter the segmented boundaries in the given input image and calculate values to enhance success rate, reduce the error rate, and compared with the existing methods.

Section 1 described the lip segmentation plays a central role in the Visual Lip Reading System. Existing methods explained in detailed and two models used in lip segmentation (i) Edge based and Region-Based models.

Section 2 described that about prior work with various segmentation methods such as ACM and LACM etc.

Section 3 proposed that the novel method used in lip segmentation. SIFT algorithm is used to extract the lip region and borderline. BPNN classification used to classify the segment area.

Section 4 explained that the result and discussion and comparative analysis with performance metrics.

Section 5 described the conclusion and complete summary of the research work.

\section{i. LITERATURE SURVEY}

Lu Y and Liu, Q et al., 2018[16] proposed research on local active contour method utilizing basic contour by linking color areas. A brilliance parallelization applied towards RGB pictures for reducing the distortion. Color space was associated that contains the U-element and mean of the two $\mathrm{d} 1$ elements and $\mathrm{d} 2$ of the film afterward transformation. Selection of the underlying parallel contour was because of the same form towards the nearest lip area. They used the semi-ellipse as the basic contour of internal and external borders.Color elements linked to acquiring the segmentation output. Performance metrics include segmentation output where comparison was done as basic contour to fragment gray picture and picture in linked color area.

Liu G and Li, H. et al., 2018 [17]studied the mass matrix development technique for the enhancement of the toughness of the contour development. Chunk matrix contains contours of the formal selection and silhouette of shape and also a standard pattern for measurement of the forms. By decreasing the standard metric restraints, active contour method implemented. Also, way prior segmented in the form of the blocks. Experimental analysis was done based on the proposed approach for improvement of the active contour method and improving the flexibility of the series of the segmented lip region image.

Wang L., Chang, Y., Wang, H., Wu Z., P u, J and Yang, X et al., 2017 [18] established a new area based contour method that depends on the two various native fixed pictures by building native hybrid picture fit power. Hybrid image decreases the modification phase for monitoring contour curvature to required borders. In this research, the proposed approach on the evaluation and comparison of various active contour methods towards segmentation of the actual pictures along with multiple strength features. The preliminary analysis does for demonstration of the performance of the exactness of the segmented

image.

Shelli L and Mengxing.al, H. et al.,2018[19]proposed research on the geometrical active contour method for removal of the deprivation issue of lower compact, fuzzy and color interference of under picture information. Picture segmentation approach was enhanced, and also a numerical method of underneath water depends on Picture segmentation approach. In this research, the experimental analysis was done based on the whole segmentation underneath water pictures by mean of lower gap and fuzzy characteristics that acquire desired segmentation output.

X u W., Y u e, X., Chen Y, and Reformat, M et al., 2017 [20] demonstrated the standard method for improvement of contour-based segmentation method. The optimum division acquired by maximization of the mass shared data among possible delivery of numerous segmented output. Performance analysis does base on the validation that collaboration of the contour basis segmentation towards partial initialization and production of the static output for contour segmented pictures.

\section{ii. RESEARCH PROPOSAL WORK}

In this section, explain the proposed work in stepwise. The Objective of the proposed work is defined as follows:

1. To study and analyze the existing methods (ACM and LACM) for Lip segmentation methods.

2. To design feature extraction using the SIFT algorithm to find the unique features, BPNN to classify the features based on crucial points.

3. To evaluate the performance parameters in the proposed framework concerning different evaluations like accuracy, Segmented Error, Overlap error, and compared with the existing (Automatic Contour) method parameters.

It has implemented the artificial intelligence classification method, and feature extraction method (SIFT) to segment and feature extract the unique properties of the lip image.

Research Methodology steps are;

1. To collect the dataset AR face Image from an online site (UCI machine learning repository) website using FACE images in .jpg format.

2. Upload the image from the dataset; convert the original image into a grayscale image (rgb2gray) format. It reduces the image dimensionality of the defined image.

3. Pre-processing phase implemented to calculate the binary image and remove the noise level in the given image. 


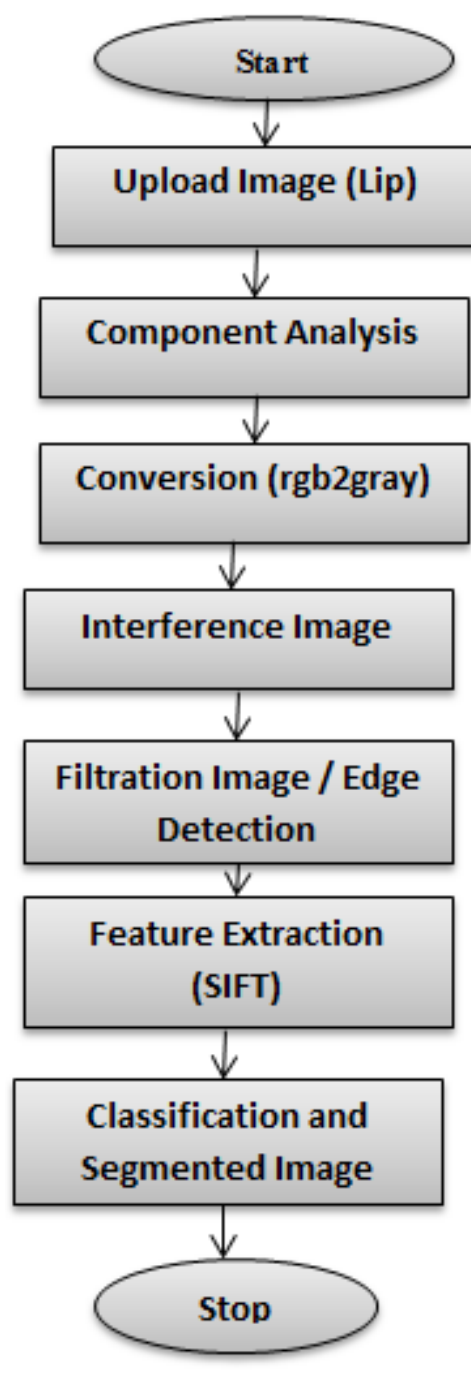

Fig. 2 Proposed Flow Chart

4. Segmentation image used to find the region of the smooth image and SIFT (Scale Invariant Feature Transformation) method used for image-based feature extraction from the input image.

5. Applying the BPNN approach on the lip image to classify and segmented image.

Compute the performance metrics and compared with the existing parameters such as accuracy rate, segmented error, and overlap error rate.

\section{i. RESULTS AND COMPARATIVE ANALYSIS}

In this section, a detailed description of the research work result screenshot, parameters metrics, and comparative analysis with proposed and existing methods.

\subsection{Result Description}

In this section, the experiment developed in MATLAB 2016a, and it has tested 200 facial images from Dataset (AR FACE).

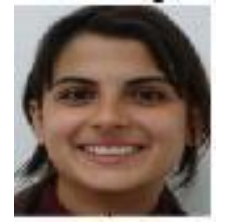

\section{Lips Cropped Image}

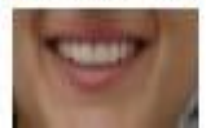

Fig. 3 (i) Real Image (ii) Cropped Image
We are applying the efficient Scale invariant with BPNN method lip region to each element and final segmented image.

Fig 3 (i) upload the input image from the original dataset. (ii) Crop the lip area from the face (AR images). In proposed work is completed by LIP SEGMENTATION.
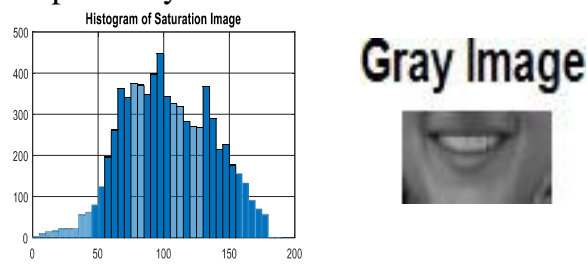

Fig. 4 (i) Histogram Image (ii) Gray Image

Fig 4 (i) shown that the Image histogram plot is a kind of image pixel values shown in a graphical pattern. If then plots the image pixels for an individual image. 4(ii) shown the grayscale image means to dimensionality reduces in a given model.
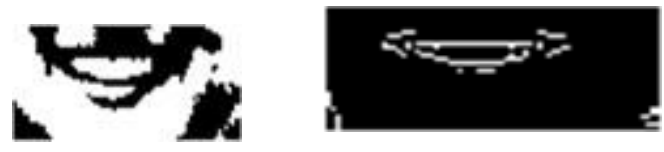

Fig. 5 (i) Binary image and (ii) Edge Image

Fig 5(i) shown that the process of Binary image dividing 2D into various binary sets and the primary goal of this image is too easy and change the representation of an image into something more meaningful and straightforward to analyses. (ii) Shown the edge detection method using a Sobel operator. It computes an approximation gradient value of the image and used image intensity function. Extract the specific points the picture, the result computes based on gradient vector based or the normalized vector function.
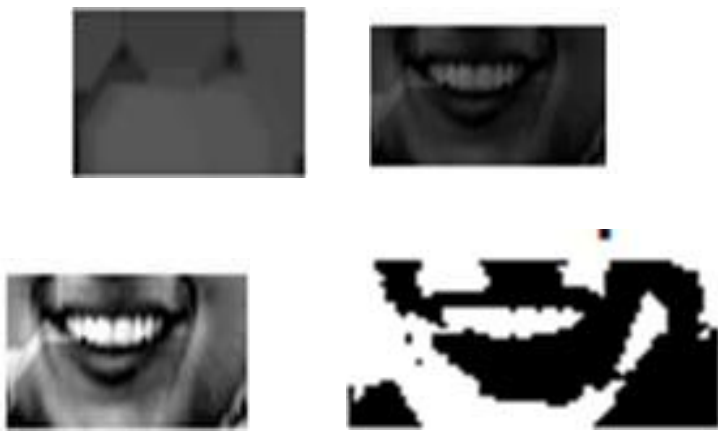

Fig. 6 (i) Background Substraction Image (ii) Approximation Image (iii) Contrast Image and (iv) Binarize Phase Image

Fig 6 (i) defines that the background image subtraction is the main data pre-processing phase in the several vision depends on applications. Fig 6(ii) defined that the approximation image with Fourier series isn't efficient because it is global and also it treats the edge or region of lip image using periodic conditions.(iii) it is the similar image luminance that creates an object differently the maximum image contrast of image is the dynamic range pattern. (iv) It shows that the clear Binary image is a digital image processing phase that has only two possible values for each image pixels. Typically, the twice color used for a Binary image is formation black and white image. The color used for the object in the image is a foreground image, while the

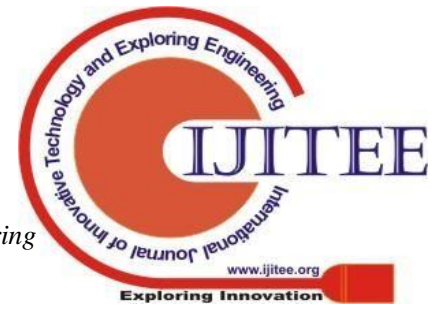


Efficient Scale invariant and Back Propagation Neural Network method using LIP Region Segmentation

rest of the image is background color.

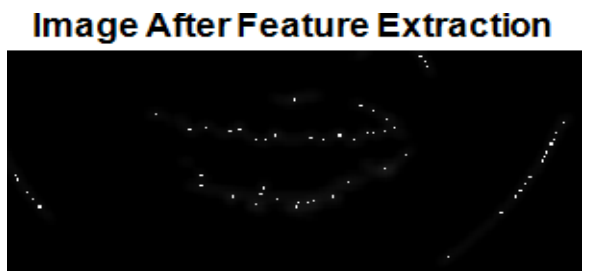

Fig. 7 Feature Extraction Test Image

Fig 7 shows the vital feature calculated by the SIFT algorithm. It localizes the feature set in the given input and evaluates the features based on unique a property which is crucial points.

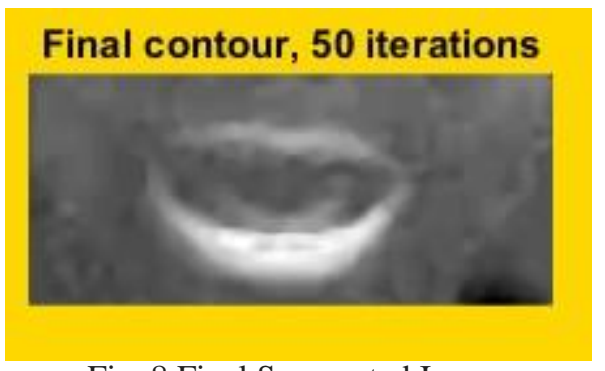

Fig. 8 Final Segmented Image

The above figure showed that the research works method divide lip area into 2 phases according to the ordinate of B1 and $\mathrm{B} 2$. If the threshold value of the ordinate difference between $\mathrm{B} 1$ and $\mathrm{B} 2$ is maximum than the $1 / 2$ height of lip image and deem the open mouth. It segments the lip image, and 50 iterations used.

Table I. Research Parameters

\begin{tabular}{|c|c|}
\hline Parameter Metrics & Values \\
\hline Overlap Error & $\mathbf{7 9 . 7}$ \\
\hline Segmentation Error & $\mathbf{1 4 . 8 6}$ \\
\hline Accuracy Rate & $\mathbf{9 4 . 6 0}$ \\
\hline MSE & $\mathbf{0 . 8 2 0}$ \\
\hline
\end{tabular}

Table II shows that the proposed research work with various parameters such as Accuracy Rate $94.60 \%$, MSE value is 0.820 , Segmented Error value is 14.86, and Overlap Error value is 14.86 .

\section{Performance Metrics}

Evaluate the accuracy performance of research algorithm; it uses two parameters [21]. The first one calculates defined as;

$$
O=\frac{a(2(B 1 \cap B 2)}{B 1+B 2} 100 \%
$$

In this eq (i) determines the overlap percentage between the segmented lip edge B1 and the ground truth B2. The second parameter is the segmented error defined as;

$$
S=\frac{O L+I L * 100}{2 * T l}
$$

Eq (ii) OL is the number of nonslip image pixel be classified (outer lip exception), ILE is the No. of lip image pixel classified as Inner lip error, and TL defines the No. of lip image pixels in the ground truth.

Accuracy is the value of the proportionality of exact classified value, which is, the sum of true positive and true negative to the sum of a true positive, true negative, false positive, and false negative. Mathematically, this stated as:

Accuracy $=\mathrm{TP}+\mathrm{TN} / \mathrm{TP}+\mathrm{TN}+\mathrm{FP}+\mathrm{FN}$.

Eq (iii) Where TP is True Positive, TN is True Negative, FP is False Positive, and FN Is false negative.

Mean Square error is the estimation of measurement of the mean of the squared errors or the difference among estimation value and estimated value.

$$
\operatorname{MSE} \frac{1}{n 1} \sum_{i=n 1+1}^{n 1+q}\left(y 1 i-Y^{\prime} 1 i\right)^{2}
$$

Eq (iv) defined that the $\left(y 1 i-Y^{\prime} 1 i\right)^{2}$ is an simple calculate quality of a particular image.

\section{Comparison Parameters}

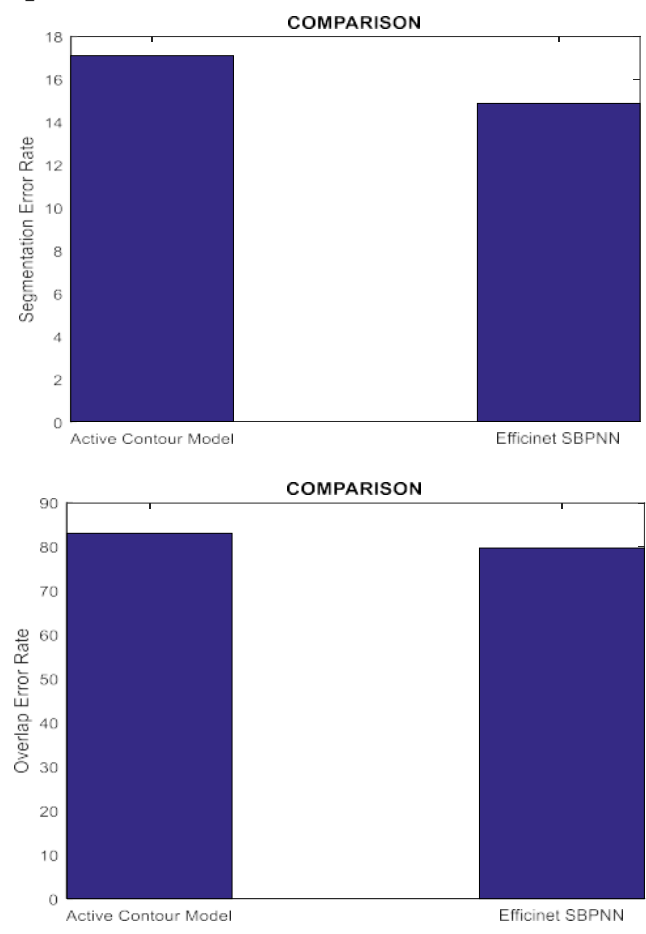

Fig 9 Comparison between proposed and existing work in segmentation and overlap error rate

Fig 9 (i) defined that the comparison between recommended and living practices with segmented Error Rate. SE is reduced rather than other parameters. (ii) Determined the correlation between proposed and existing works by Overlap Error Rate. It OER is diminished rather than in different settings.

Table II Comparison Between proposed and existing Methods

\begin{tabular}{|c|c|c|}
\hline $\begin{array}{c}\text { Parameter } \\
\text { Metrics }\end{array}$ & $\begin{array}{c}\text { Proposed } \\
\text { Work }\end{array}$ & $\begin{array}{c}\text { Existing } \\
\text { Work }\end{array}$ \\
\hline Overlap Error & $\mathbf{7 9 . 7 3}$ & $\mathbf{8 3 . 1 0}$ \\
\hline
\end{tabular}




\begin{tabular}{|c|c|c|}
\hline $\begin{array}{c}\text { Segmentation } \\
\text { Error }\end{array}$ & 14.869 & 17.10 \\
\hline
\end{tabular}

Table II defined the comparison between proposed and existing work with segmentation and overlap error rate and reduced the error rate and high the accuracy rate.

\section{i. CONCLUSION AND FUTURESCOPE}

A Lip segmentation technique based active contour segmentation that used for lip reading scheme. Picture analysis and the computer system are done through a dynamic contour model. In lip image segmentation, contours are achieved by acquiring the class borders by minimization of the fixed power. Hence, the geometrical contour model is complex than the active contour method. For multiple images, it is not easy to prepare contour curvature. To overcome this issue differentiated various segmentations receive a high-grade lip image segmentation. In research work, the proposed method implemented to achieve a better accuracy rate and segmented results and compared with the existing process using area or circle as the region to segment grayscale images and combined with the color-space image. The main advantages of this SIFT and BPNN method of this technique the results of lips because the inner region found. In proposing a method implemented accuracy achieved $94 \%$, and the error rate reduces with Segmented Error $14.1 \%$ and Overlap Error rate value is $79.73 \%$.

In future work can implement Filtration and contour methods to detect the region points and achieve the image quality parameter also (PSNR) and RMSE (Root Mean Square Error Rate).

\section{REFERENCES}

1. Chin, S. W., Seng.al, K. P., Ang.al, L. M. and Lim, K. H. (2009, March), "New lips detection and tracking system.,,In Proceedings of the international multi conference of engineers and computer scientists, Vol. 1, pp. 18-20.

2. Sadaghiani.al, M. H., Seng.al, K. P., Chin, S. W., and Ang.al, L. M. (2009, November),'Enhanced Lips Detection and Tracking System" ,In International Visual Informatics Conference (pp. 254-265).Springer, Berlin, Heidelberg.

3. Karlsson.al, S. M., and Bigun.al, J. (2012, June), "Lip-motion events analysis and lip segmentation using optical flow" "In 2012 IEEE Computer Society Conference on Computer Vision and Pattern Recognition Workshops (pp. 138-145).IEEE.

4. Yan, X. P., Li, X. Q., Zheng.la, L. L and Li, F. F. (2010, September),"Robust lip segmentation method based on the level set model" , In Pacific-Rim Conference on Multimedia (pp. 731739).Springer, Berlin, Heidelberg.

5. Wang, S. L., Lau, W. H., Liew.al, A. W. C and Leung, S. H. (2007).,'Robust lip region segmentation for lip images with complex background"' Pattern Recognition, vol.40(12), pp.3481-3491.

6. Munot.al, K., Mehta, N., Mishra.la, S. and Chaturvedi.la, R. N. (2017),"Areview on image segmentation techniques with an application perspective.",International Journal of Advanced Research in Computer Science, vol. 8(9).

7. Li, M. and Cheung, Y. M. (2010, September),"Automatic segmentation of color lip images based on morphological filter", In International Conference on Artificial Neural Networks (pp. 384387).Springer, Berlin, Heidelberg.

8. Kalbkhani.al, H. and Amirani.al, M. C. (2012),"An efficient algorithm for lip segmentation in color face images based on local information", Journal of World's Electrical engineering and technology, vol.l(1), 2012.

9. Naz.al, S., Majeed.al, H and Irshad.al, H. (2010, October). Image segmentation using fuzzy clustering: A survey. In 2010 6th international conference on emerging technologies (ICET) (pp. 181186).IEEE.

10. Liew.al, A. C., Leung, S. H and Lau, W. H. (2003)," Segmentation of color lip images by spatial fuzzy clustering",IEEE transactions on Fuzzy Systems, vol 11(4), pp 542-549.

11. Zhang, D and Lu, G. (2001),"Segmentation of moving objects in image sequence: A review", Circuits, Systems and Signal Processing, vol 20(2), pp 143-183.

12. Stillittano.al, S and Caplier.al, A. (2008, January),'Inner Lip Segmentation by Combining Active Contours and Parametric Models", In VISAPP (1) (pp. 297-304).

13. Lu, Y and Zhou, T. (2018), "Lip segmentation using localized active contour model with automatic initial contour", Neural Computing and Applications, 29(5), 1417-1424.

14. Liévin.al, M., Delmas.al, P., Coulon.al, P. Y., Luthon.al, F and Fristol.al, V. (1999, July)," Automatic lip tracking: Bayesian segmentation and active contours in a cooperative scheme", In Proceedings IEEE International Conference on Multimedia Computing and Systems (Vol. 1, pp. 691-696).IEEE.

15. Saeed.al, U and Dugelay.al, J. L. (2010, July),"Combining edge detection and region segmentation for lip contour extraction “,In International Conference on Articulated Motion and Deformable Objects (pp. 11-20).Springer, Berlin, Heidelberg.

16. Lu, Y and Liu, Q. (2018),'Lip segmentation using automatic selected initial contours based on localized active contour model ",EURASIP Journal on Image and Video Processing, 201 vol 8(1), 7.

17. Liu, G., and Li, H. (2018)," Robust evolution method of active contour models and application in segmentation of image sequence.",Journal of Electrical and Computer Engineering, 2018.

18. Wang, L., Chang, Y., Wang, H., Wu, Z., Pu, J and Yang, X. (2017),' An active contour model based on local fitted images for image segmentation," Information sciences, vol. 418,pp. 61-73.

19. Shele.ali, L. and Mengxing.al, H. (2018, March).,"Research of Underwater Image Segmentation Algorithm Based on the Improved Geometric Active Contour Models", In 2018 International Conference on Intelligent Autonomous Systems (ISO IAS) (pp. 44-50).IEEE.

20. X u, W., Y u e, X., Chen, Y and Reformat, M. (2017, September),'Ensemble of active contour based image segmentation", In 2017 IEEE International Conference on Image Processing (ICIP) (pp. 86-90).IEEE.

21. Zaman.al, M. and Lung, C. H. (2018, April).,"Evaluation of machine learning techniques for network intrusion detection",In NOMS 20182018 IEEE/IFIP Network Operations and Management Symposium (pp. 1-5).IEEE.

\section{AUTHORS PROFILE}

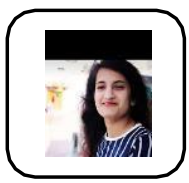

Shaifali Sharma received my B.Tech degree in Computer Science And Engineeering From Indore Institute Of Science And Technology,Indore Madhaya Pradesh ,India 2017. I m currently M.Tech student at Chandigarh Engineering College Landran ,Mohali Punjab, india in the department of Computer Science \& Engineering Since 2017 . my area of intrest are Artifitial Intelligence

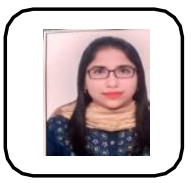

Geetanjali Babbar,Working as an Assistant Professor in Chandigarh Engineering College, Landran, Pursuing $\mathrm{Ph} . \mathrm{D}$ in Computer Science, M.Tech in Computer Science, 16 national/international publications, Life time member of Indian Society for Technical Education. I have 15 years of teaching experience in CEC Landran. I have been awarded for best teacher in computer science department of CEC Landran. My research work is in the area of Machine Learning.

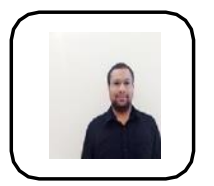

Ishdeep Singla has born sangrur,punjab,sangrur in 1986 ,he revceived master of engineering degree from Thapar university, patiala in software engineering stream and B.Tech degree in computr engineering steam from punjabi university, patiala his area of interest is biomedical engineering, data science and machine learning 
Efficient Scale invariant and Back Propagation Neural Network method using LIP Region Segmentation

Dr. Gagandeep received his Bachelor's degree in Computer Science and Engineering from Punjab Technical University, Jalandhar, Punjab, India in 2002, M.E. degree in Computer Science and Engineering from PEC University of Technology, Chandigarh, India, in 2005 and Ph.D. degree in Computer Engineering from Panjabi university, Patiala, India, in 2017. Presently, he works in Department of Computer Science \& Engineering, Chandigarh Engineering College (CEC), Landran, Mohali, India as faculty member and has several years work experience in the areas of teaching. He has published more than 40 research papers in leading journals, conference proceedings and books including Elsevier, IEEE, Taylor n Francis and Springer and also holds 23 patents. He is also life member of several societies, received prestigious Academia award from CSI consecutively in $2011 \& 2012$ and also received ISTE best teacher award in 2016. His current research interests include medical image processing, object detection, semantic retrieval, deep learning 